\title{
A commonly occurring genetic variant within the NPLOC4-TSPAN10-PDE6G gene cluster is associated with the risk of strabismus
}

\author{
Denis Plotnikov ${ }^{1} \cdot$ Rupal L. Shah ${ }^{1}$. Jamille N. Rodrigues ${ }^{2}$. Phillippa M. Cumberland ${ }^{3,4}$ • Jugnoo S. Rahi ${ }^{3,4,5,6}$.

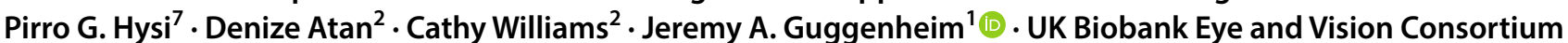

Received: 20 December 2018 / Accepted: 20 April 2019 / Published online: 9 May 2019

(c) The Author(s) 2019

\begin{abstract}
Strabismus refers to an abnormal alignment of the eyes leading to the loss of central binocular vision. Concomitant strabismus occurs when the angle of deviation is constant in all positions of gaze and often manifests in early childhood when it is considered to be a neurodevelopmental disorder of the visual system. As such, it is inherited as a complex genetic trait, affecting $2-4 \%$ of the population. A genome-wide association study (GWAS) for self-reported strabismus (1345 cases and 65,349 controls from UK Biobank) revealed a single genome-wide significant locus on chromosome 17q25. Approximately 20 variants across the NPLOC4-TSPAN10-PDE6G gene cluster and in almost perfect linkage disequilibrium (LD) were most strongly associated (lead variant: $\mathrm{rs} 75078292, \mathrm{OR}=1.26, p=2.24 \mathrm{E}-08$ ). A recessive model provided a better fit to the data than an additive model. Association with strabismus was independent of refractive error, and the degree of association with strabismus was minimally attenuated after adjustment for amblyopia. The association with strabismus was replicated in an independent cohort of clinician-diagnosed children aged 7 years old (116 cases and 5084 controls; $\mathrm{OR}=1.85, p=0.009$ ). The associated variants included 2 strong candidate causal variants predicted to have functional effects: rs6420484, which substitutes tyrosine for a conserved cysteine (C177Y) in the TSPAN10 gene, and a 4-bp deletion variant, rs397693108, predicted to cause a frameshift in TSPAN10. The population-attributable risk for the locus was approximately 8.4\%, indicating an important role in conferring susceptibility to strabismus.
\end{abstract}

Electronic supplementary material The online version of this article (https://doi.org/10.1007/s00439-019-02022-8) contains supplementary material, which is available to authorized users.

Cathy Williams

cathy.williams@bristol.ac.uk

Jeremy A. Guggenheim

GuggenheimJ1@cardiff.ac.uk

1 School of Optometry and Vision Sciences, Cardiff University, Cardiff CF24 4HQ, UK

2 Population Health Sciences, Bristol Medical School, University of Bristol, 1-5 Whiteladies Road, Bristol BS8 1NU, UK

3 Life Course Epidemiology and Biostatistics Section, Institute of Child Health, University College London, London WC1N 1EH, UK

\section{Introduction}

Strabismus refers to an abnormal alignment of the eyes leading to loss of binocular vision. Concomitant strabismus occurs when the angle of deviation is constant in all positions of gaze and often manifests in early childhood when it is considered to be a neurodevelopmental disorder of the visual system.

4 Ulverscroft Vision Research Group, University College London Institute of Child Health, London WC1N 1EH, UK

5 University College London Great Ormond Street Institute of Child Health, London WC1N 3JH, UK

6 National Institute for Health Research Biomedical Research Centre at Moorfields Eye Hospital NHS Foundation Trust and University College London Institute of Ophthalmology, London WC1E 6BT, UK

7 Department of Twin Research and Genetic Epidemiology, King's College London, St Thomas' Hospital, London SE1 7EH, UK 
Concomitant strabismus is most often convergent ('esotropia') or divergent ('exotropia'), although vertical misalignment may also occur as the primary deviation or in conjunction with esoor exotropia. It is often associated with amblyopia (defined as poor visual acuity in one or both eyes not immediately correctable by glasses and without accompanying ocular pathology) in the deviated eye-either because the squint itself leads to secondary amblyopia in the deviated eye or because reduced vision in one eye compared with the other (e.g. anisometropia, unilateral cataract) secondarily leads to the squint. Therefore, concomitant strabismus is frequently associated with childhood-onset visual impairment, usually in one eye (Robaei et al. 2006).

A variety of prenatal and early life environmental factors, such as prematurity, maternal smoking, and ill-health during pregnancy, increase the risk of strabismus, as does a high hyperopic refractive error in early childhood (Cotter et al. 2011; Pathai et al. 2010; Atkinson et al. 1996). Numerous studies have been conducted to understand the genetics of strabismus (Graeber et al. 2013; Kruger et al. 2013; Maconachie et al. 2013). Despite a complex inheritance pattern (Sanfilippo et al. 2012; Ye et al. 2014; Georges et al. 2013), linkage analysis in pedigrees with multiple affected participants has implicated a locus at $7 \mathrm{p} 22.1$ (strabismus, susceptibility to, 1 ; STBMS1) consistent with the idea that rare, monogenic subtypes may exist (Parikh et al. 2003; Rice et al. 2009). Strabismus is also a feature of several rare syndromes-often in conjunction with intellectual disability_including examples such as Mietens-Weber syndrome and Lamb-Shaffer syndrome.

Recently, Shaaban et al. (2018) tested the hypothesis that commonly occurring polymorphisms in the general population increase the risk of non-syndromic strabismus, by carrying out a genome-wide association study (GWAS) for strabismus. They identified a locus within the first intron of WRB (tryptophan-rich basic protein) on chromosome 21 (lead variant $\mathrm{rs} 2244352 ; \mathrm{OR}=1.33, p=9.58 \mathrm{E}-11$ ). Here, we sought to test the same hypothesis in a larger sample, in order to increase statistical power. In view of the known association between strabismus and hyperopia, the refractive error of participants was included as a covariate in our GWAS analysis to avoid detecting genetic variants that increase the risk of strabismus secondary to hyperopia. The advent of the UK Biobank project facilitated this work, by providing a larger sample of cases with strabismus than has been studied before.

\section{Materials and methods}

\section{Phenotypes in UK Biobank participants}

Approximately 500,000 individuals aged $37-73$ years were recruited into the UK Biobank cohort study between
February 2006 and July 2010 (Sudlow et al. 2015). Ethical approval was obtained from the National Health Service National Research Ethics Service (Ref 11/NW/0382) and all participants provided written informed consent. At a baseline visit to 1 of 22 assessment centres across the UK, participants completed a touch-screen questionnaire and underwent a series of physical assessments. From 2009, the physical assessments included an ophthalmic examination (Cumberland et al. 2016). Approximately 23\% of participants underwent the ophthalmic assessment at the baseline visit, while an additional small proportion completed this assessment at the first of up to two follow-up visits. Based on their reason for wearing spectacles or contact lenses at the baseline or first follow-up visit [field \#6147], participants were classified as having self-reported strabismus. Specifically, participants were asked 'Why were you prescribed glasses/contact lenses? (You can select more than one answer)'. The response options were: (1) 'For short-sightedness, i.e. only or mainly for distance viewing such as driving, cinema, etc., (called "myopia")'; (2) 'For long-sightedness, i.e. for distance and near, but particularly for near tasks like reading (called "hypermetropia")'; (3) 'For just reading/near work as you are getting older (called "presbyopia")'; (4) 'For "astigmatism"; (5) "For a "squint" or "turn" in an eye since childhood (called "strabismus")'; (6) 'For a "lazy" eye or an eye with poor vision since childhood (called "amblyopia")'; (8) 'Other eye condition', or (7) 'Do not know'. Note that the age-of-onset, and the type of strabismus, for example esotropia or exotropia, was not ascertained during the UK Biobank assessment, hence all subtypes would have been captured within the 'self-reported strabismus' phenotype. Similarly, information on strabismus surgery in childhood was not available (since data on hospital in-patient operations were collected only from April 1997 onwards). In addition, participants who had strabismus, but who were not prescribed glasses/contact lenses because of the condition, would have been categorised as 'controls' rather than 'cases' for this self-reported strabismus phenotype.

Non-cycloplegic refractive error was measured after removal of habitual spectacles or contact lenses using a Tomey RC5000 autorefractor [fields \#5084, \#5085, \#5086 and \#5087]. All repeat readings were averaged after removal of those flagged as unreliable [fields \#5090 and \#5091]. Spherical equivalent refractive error was calculated as sphere power plus half the cylinder power. The refractive error of an individual was taken as the average spherical equivalent of the two eyes (Tedja et al. 2018). Myopia was defined as a spherical equivalent refractive error $\leq-0.50$ diopters (D). Refractive astigmatism was taken as the average cylinder power between the two eyes. A binary variable was used to classify individuals with astigmatism $\geq 1.00 \mathrm{D}$ (Shah et al. 2018). Anisometropia was calculated as the difference in spherical equivalent between the two eyes. A 
binary variable was used to classify individuals with anisometropia $\geq 1.00 \mathrm{D}$ (Qin et al. 2005).

\section{Genetic data in UK Biobank participants}

UK Biobank carried out DNA extraction from blood samples, and obtained high-density genotypes using either the UK BiLEVE array $(n=49,950)$ or the UK Biobank Axiom array $(n=438,427)$. Prior to release of the genetic data ("July 2017 release"), Bycroft et al. (2018) performed extensive quality control of the genotype data and imputed additional genotypes using the $\mathrm{HRC}$ reference panel (McCarthy et al. 2016). Imputed genotype data were available for 488,377 individuals. Participants who had withdrawn consent were removed, as were those not in the White British European ancestry subset defined by Bycroft et al. (2018), those whose genetic sex did not match their self-reported gender, and those with heterozygosity more than 4 standard deviations from the mean. A set of well-imputed variants (with IMPUTE2 INFO metric $>0.9$, MAF $>0.005$, missing rate $\leq 0.01$, and an 'rs' variant ID prefix) were selected and LD-pruned using the --indep-pairwise 5050.1 command in PLINK 2.0 (Chang et al. 2015). These were used to create a genetic relationship matrix (GRM) with PLINK 2.0 in order to identify a set of unrelated individuals (command --relcutoff 0.025 ). Note that phenotype information was not taken into account when selecting a set of unrelated participants. This left 338,253 unrelated participants of White British ancestry.

As shown in Fig. 1, further exclusions were made for participants who: did not have a response for the questionnaire item [field \#6147] 'Why were you prescribed glasses/contact lenses?'; did not have valid autorefraction information; selfreported a history of laser refractive surgery [field \#5325], cataract surgery [field \#5324; \#20004; ICD 1435], corneal graft surgery [field \#5328], any other eye surgery in the last 4 weeks [field \#5181], any eye trauma resulting in sight loss [\#5419, \#6148], serious eye problems [field \#6148], or selfreport of having cataracts [fields \#5441, \#6148; \#20004; ICD 1278] or retinal detachment [field \#20004; ICD 1281]. Participants were also excluded if their hospital records [field \#41200] indicated they had undergone cataract surgery [OPCS-4: C711, C712, C718, C719, C723, C751], retinal detachment surgery [OPCS-4: C548, C549, C543, C545], or corneal surgery [OPCS-4: C442, C444, C445, C448, C461, C462, C463, C465, C493]. The final UK Biobank 'discovery sample' comprised 66,694 participants.

\section{Genome-wide association study for strabismus in UK Biobank participants}

A GWAS for self-reported strabismus was carried out for the 1345 cases and 65,349 controls in the discovery sample.



Fig. 1 Flow diagram illustrating the selection of UK Biobank participants for the GWAS sample

Firth logistic regression was performed (--glm-firth command in PLINK 2.0) for 7,469,170 imputed variants in the $\mathrm{HRC}$ reference panel with $\mathrm{MAF} \geq 1 \%$, INFO $>0.8$, missing genotype rate $<1.5 \%$, and HWE $p$ value $>1.0 \mathrm{e}-06$. Only individuals with a missing genotyping rate $<2.5 \%$ were included. Age, gender, refractive error averaged between the two eyes, genotyping array (UK BiLEVE or UK Biobank Axiom, coded as a binary variable), and the first 10 ancestry PCs were included as covariates.

\section{Post-GWAS analyses}

Fine-mapping of the NPLOC4-TSPAN10-PDE6G region was carried out using FINEMAP v1.3 (Benner et al. 2016). 
All SNPs on the HRC reference panel within $\pm 250 \mathrm{~kb}$ of the lead variant rs75078292 or that were in $\operatorname{LD}\left(r^{2}>0.1\right)$ with the lead variant were included in the analysis. Up to 5 causal variants in the region were considered, with a prior probability within the range $0.583-0.005$ of having $1-5$ causal variants. The Genotype-Tissue Expression consortium (GTEx) Portal (The GTEx Consortium 2015) was accessed on $09 / 18 / 2018$ to identify expression quantitative trait loci (eQTL) for genes situated at the locus regulated by the lead SNP identified in the GWAS (so-called 'eGenes').

\section{Statistical analyses and population-attributable risk}

Statistical tests were performed using R (R Development Core Team 2008). Standard logistic regression models were fitted using the $\mathrm{glm}$ function, while Firth regression models were fitted using the brglm function from the brlrmr package. A likelihood ratio test was used to compare the fit of additive vs. dominant or additive vs. recessive models (Bagos 2013). This was accomplished by deriving dummy variables and fitting nested models, as described in Online Resource 1. In view of the low prevalence (2-4\%) of strabismus, we made the assumption that the relative risk was approximately equal to the odds ratio (OR), and therefore the population-attributable risk (PAR) due to the lead GWAS variant was estimated with the formula (Qi et al. 2011): $\mathrm{PAR}=p(\mathrm{OR}-1) /[p(\mathrm{OR}-1)]+1$, where $p$ is the proportion of controls with the risk genotype. The IMPUTE2 INFO score for the lead GWAS variants rs75078292 was 0.999 , suggesting that there was no loss in accuracy by using 'hard' genotype calls in the above models rather than dosage accounting for genotype uncertainty.

\section{ALSPAC replication sample}

The Avon Longitudinal Study of Parents and Children (ALSPAC) (Boyd et al. 2013; Fraser et al. 2012) is a birth cohort study that recruited 14,541 pregnant women resident in Avon, UK, with expected dates of delivery 01/04/1991-31/12/1992. Of these initial pregnancies, 13,988 children were alive at 1 year of age. When the oldest children were approximately 7 years of age, an attempt was made to bolster the initial sample with eligible cases who had failed to join the study originally. This resulted in an additional 811 children joining the study. Ethical approval for the study was obtained from the ALSPAC Ethics and Law Committee and the Local Research Ethics Committees. Please note that the study website (http://www.bristol.ac.uk/alspac/researcher s/our-data/) contains details of all the data that are available through a fully searchable data dictionary and variable search tool.

DNA extraction, genotyping and imputation of ALSPAC participants has been described previously (Taylor et al.
2016). Imputed genotype data were available for a total of 8237 children. Eye movements were assessed by an orthoptist when ALSPAC participants were aged 7 years old (Williams et al. 2008). Ocular misalignment was quantified by simultaneous prism cover test and alternate prism cover test, at near $(33 \mathrm{~cm})$ and distance $(6 \mathrm{~m})$ with and without glasses, if worn. Strabismus was classified as 'manifest' if present in normal viewing with both eyes open. Horizontal strabismus was categorised as esotropia (convergent) or exotropia (divergent) and included manifest and large latent deviations ( $>10 \mathrm{pd}$ for convergent and $>15 \mathrm{pd}$ for divergent) so as to be more likely to capture intermittent or decompensating cases. Logistic regression was used to examine the association between each ocular phenotype and rs6420484 genotype (coded either as additive or recessive) in the 5200 children with genotype and ocular phenotype data available. The phenotypes examined were: parentally reported history of strabismus ( $n=145$ cases), manifest strabismus ( $n=116$ cases), esotropia ( $n=143$ cases) and exotropia ( $n=28$ cases).

\section{Retinal immunohistochemistry}

Immunostaining of adult mouse retinal sections ( $>8$ weeks old) was performed as previously described (Jung et al. 2015). Antibodies were used at the following dilutions: peanut agglutinin conjugated to Alexa 488 (1:500), mouse antirhodopsin (1:5000), rabbit anti-TSPAN10 (1:1000), mouse anti-GOalpha (1:500), rabbit NPLOC4 (1:10), and mouse anti-PKC (1:1000). Images were acquired using a Leica laser scanning confocal microscope.

\section{Results}

\section{Validation of self-reported strabismus}

There were 72,911 unrelated UK Biobank participants who reported their country of birth as England, Wales or Scotland, whose genetic ancestry principal components clustered with other White British Europeans, and who had responded to the questionnaire item 'Why were you prescribed glasses/ contact lenses? (You can select more than one answer)' as well as undergoing an autorefraction assessment. After further removal of participants with a history of ocular pathology, this left 66,694 individuals (Fig. 1). A total of 1345 (2.0\%) of participants reported 'squint or a turn in an eye since childhood' as a reason for wearing glasses or contact lenses. Participants with self-reported strabismus had a 11.3-fold greater prevalence of self-reported amblyopia, a 2.5 -fold greater prevalence of $1.00 \mathrm{D}$ or more anisometropia, a much more hypermetropic refractive error (median +2.46 vs. $+0.21 \mathrm{D}$ ), and a much earlier age of starting to wear glasses or contact lenses (median 5 vs. 40 years old) 
(Table 1; Fig. 2). For age and gender, the cases and controls were well-matched (Table 1), and the difference in Townsend Deprivation Index, a measure of socioeconomic position, in those who did vs. did not report having strabismus was modest ( -1.84 vs $-2.12 ; p=4.40 \mathrm{E}-03)$. All of these comparisons between cases and controls supported the validity of the self-reported strabismus phenotype in the majority of participants.

\section{Genome-wide association study for self-reported strabismus identifies a locus on 17q25.3}

Standard logistic regression is susceptible to bias in highly unbalanced case-control studies, which can result in either conservative or anti-conservative test behaviour (Ma et al. 2013). We therefore carried out our GWAS analysis using Firth bias-corrected logistic regression. A total of 7,469,170 genetic variants were tested for association with selfreported strabismus in the sample of 1345 cases and 65,349 controls (Fig. 1). As well as standard GWAS covariates such as age, sex and PCs, we included refractive error averaged between the two eyes as an additional covariate in order to mitigate against identifying genetic variants primarily associated with this comorbid trait.

Variants located on chromosome 17q25.3 were found to be significantly associated with strabismus (Fig. 3). The most strongly associated variant was rs75078292, OR $=1.26$, 95\% CI 1.16-1.36, $p=2.24 \mathrm{E}-08$. Markers in other regions did not attain genome-wide significance; the lead variant in all regions that include a marker with $p<1.0 \mathrm{E}-05$ are listed in Online Resource 2. The strabismus GWAS analysis showed no evidence of population stratification $\left(\lambda_{\mathrm{GC}}=1.004\right.$; Online Resource 3).

The lead variant, rs2244352, identified in the non-accommodative esotropia case-control GWAS reported by Shaaban et al. (2018) was not associated with self-reported strabismus in the current GWAS (OR $=1.01,95 \%$ CI $0.93-1.10$, $p=0.83$ ).

\section{Fine-mapping of the $17 q 25.3$ locus}

Conditional analysis suggested that a single variant in the region was driving the association at the NPLOC4-TSPAN10-PDE6G locus (Fig. 4). To investigate this further, we used the FINEMAP Bayesian fine-mapping approach developed by Benner et al. (2016), which exploits a shotgun stochastic search algorithm to greatly increase computational performance when evaluating the evidence for multiple causal variants in a genomic region. However, this analysis was not able to infer the precise causal variant(s) from amongst the approximately 20 variants in almost perfect LD. Since the associated variants at the locus have widely varying MAF across ancestry groups (Fig. 5), future fine-mapping efforts may be more successful in populations of non-European ancestry.

\section{Mode of inheritance attributable to the NPLOC4- TSPAN10-PDE6G locus}

We explored if an additive, dominant or recessive model best described the relationship between rs75078292 genotype and strabismus. An additive model provided a better fit than a dominant model ( $p=4.76 \mathrm{E}-06)$ while a recessive model provided a better fit than the additive model $(p=8.10 \mathrm{E}-05)$. The same pattern of results was evident after including the presence/absence of amblyopia, refractive error, astigmatism, and/or anisometropia as covariates in the analysis (Table 2).

Under the recessive model, the risk of strabismus in the $14.8 \%$ of the sample homozygous for the minor

Table 1 Demographic and ocular characteristics of the UK Biobank strabismus GWAS sample

\begin{tabular}{|c|c|c|c|c|c|}
\hline Variable & & All $(n=66,694)$ & $\begin{array}{l}\text { Cases (self-reported } \\
\text { strabismus; } n=1345 \text { ) }\end{array}$ & Controls $(n=65,349)$ & $p$ value \\
\hline Female & $N(\%)$ & $35,758(53.6 \%)$ & $803(59.7 \%)$ & $34,955(53.5 \%)$ & $2.20 \mathrm{E}-01$ \\
\hline Self-reported unilateral amblyopia & $N(\%)$ & $2465(3.7 \%)$ & $471(35.0 \%)$ & $1994(3.1 \%)$ & $<1.0 \mathrm{E}-99$ \\
\hline Both eyes VA $\leq 0.0 \log$ MAR & $N(\%)$ & $30,098(45.1 \%)$ & $266(19.8 \%)$ & $29,832(45.6 \%)$ & $<1.0 \mathrm{E}-99$ \\
\hline VA difference $\geq 0.2 \log$ MAR & $N(\%)$ & $13,135(19.7 \%)$ & $624(46.4 \%)$ & $12,511(19.1 \%)$ & $<1.0 \mathrm{E}-99$ \\
\hline Better VA $\leq 0.0$; VA difference $\geq 0.2$ & $N(\%)$ & $9123(13.7 \%)$ & $454(33.8 \%)$ & $8669(13.3 \%)$ & $<1.0 \mathrm{E}-99$ \\
\hline Anisometropia $\geq 1.0 \mathrm{D}$ & $N(\%)$ & $11,156(16.7 \%)$ & $553(41.1 \%)$ & $10,603(16.2 \%)$ & $<1.0 \mathrm{E}-99$ \\
\hline Anisometropia $\geq 2.0 \mathrm{D}$ & $N(\%)$ & $3948(5.9 \%)$ & $261(19.4 \%)$ & $3687(5.6 \%)$ & $<1.0 \mathrm{E}-99$ \\
\hline Age & Median (IQR) & $60.17(53.33$ to 64.42$)$ & $60.17(53.67$ to 64.83$)$ & $60.17(53.33$ to 64.42$)$ & $2.30 \mathrm{E}-01$ \\
\hline Refractive error (D) average of 2 eyes & Median (IQR) & $0.23(-1.41$ to 1.26$)$ & $2.46(0.52$ to 4.65$)$ & $0.21(-1.44$ to 1.22$)$ & $<1.0 \mathrm{E}-99$ \\
\hline Anisometropia (D) & Median (IQR) & $0.36(0.16$ to 0.73$)$ & 0.75 (0.30 to 1.67$)$ & $0.35(0.15$ to 0.72$)$ & $8.00 \mathrm{E}-94$ \\
\hline Age started wearing glasses (years) & Median (IQR) & $39.00(15.00$ to 47.00$)$ & $5.00(3.00$ to 8.00$)$ & $40.00(16.00$ to 47.50$)$ & $<1.0 \mathrm{E}-99$ \\
\hline Townsend Deprivation Index & Median (IQR) & $-2.12(-3.59$ to 0.25$)$ & $-1.84(-3.46$ to 0.63$)$ & $-2.12(-3.59$ to 0.25$)$ & $4.40 \mathrm{E}-03$ \\
\hline
\end{tabular}


Fig. 2 Clinical and demographic characteristics of participants self-reporting strabismus compared to controls ( $n=1345$ cases and $n=65,349$ controls). Early age-of-onset of glasses was defined as $\leq 7$ years. Bar chart error bars denote $95 \%$ CI. For violin plots, the white rectangle corresponds to the interquartile range and the solid black circle to the median
Female (\%)

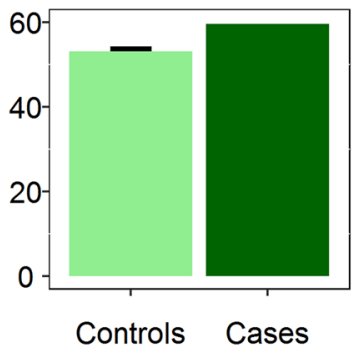

Amblyopia (\%)

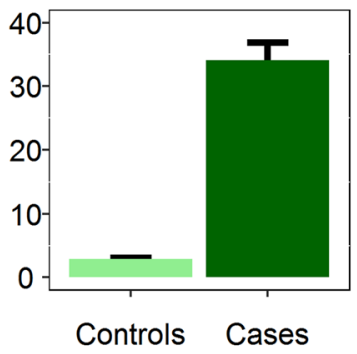

Refractive error (D)

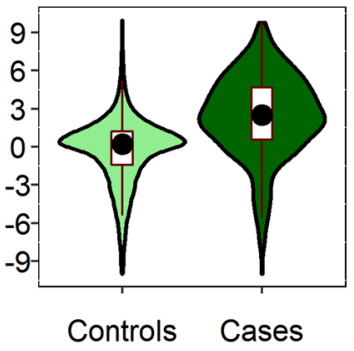

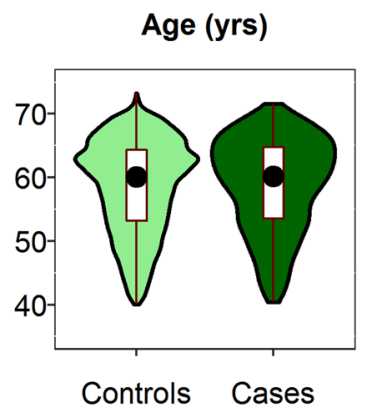

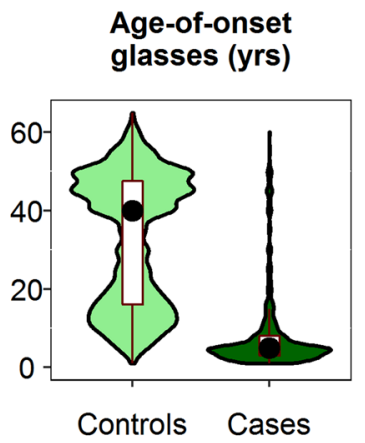

Early age-of-onset glasses (\%)

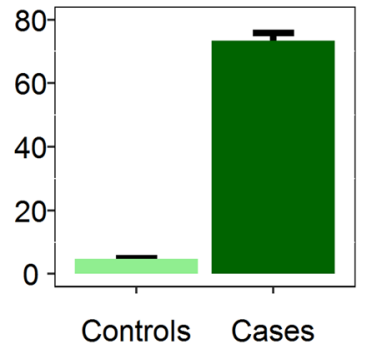

Townsend Deprivation Index

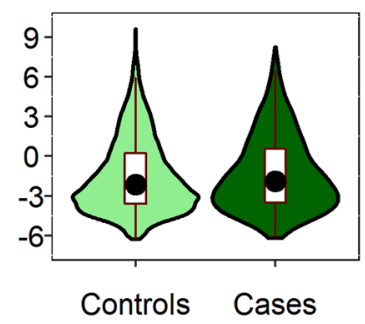

Anisometropia 1.00 D (\%)

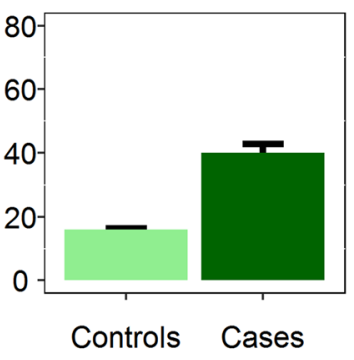

Anisometropia (D)

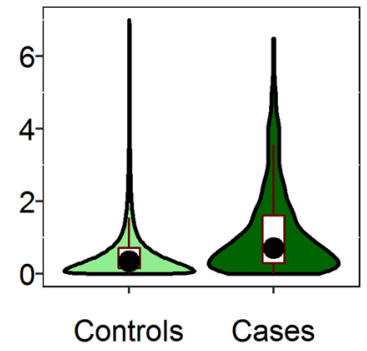

allele of rs75078292 was OR $=1.62$ (95\% CI 1.41-1.86, $p=1.01 \mathrm{E}-11)$. This suggested that the population-attributable risk due to the locus was approximately $8.4 \%$.

\section{Co-localisation of association signals for strabismus and refractive error}

The NPLOC4-TSPAN10-PDE6G locus was identified in a previous GWAS for self-reported myopia by Pickrell et al. (2016) carried out using data for individuals of European ancestry from the personal genomics company 23 andMe. The variant most strongly associated with refractive error in the Pickrell et al. study was rs9747347, which is in perfect LD with the lead strabismus variant $\left(r^{2}=1.00\right)$. In analyses adjusted for age, sex, genotyping array and the first 10 PCs, we found that the strabismus risk allele of rs75078292 was also associated with myopia $(\mathrm{OR}=1.06$, $p=5.78 \mathrm{E}-07$ ) and refractive error (regression coefficient $=-0.09 \mathrm{D}, 95 \% \mathrm{CI}-0.12$ to -0.06 , per copy of the risk allele, $p=2.77 \mathrm{E}-08$ ) in UK Biobank participants consistent with the perfect LD between rs9747347 and the similar frequencies of their respective risk alleles. Adjusting for amblyopia or strabismus had minimal impact on the magnitude of the association between rs75078292 and either myopia or refractive error.

After stratifying the sample into myopic, emmetropic and hyperopic subsamples (Table 3), there was no longer evidence of an association between rs75078292 genotype and strabismus in the myopic stratum $(\mathrm{OR}=1.11, p=6.19 \mathrm{E}-01)$, whereas the association in the hyperopic stratum remained strong $(\mathrm{OR}=1.64, p=8.30 \mathrm{E}-07)$. Association was evident in emmetropes $(\mathrm{OR}=1.88, p=7.24 \mathrm{E}-03)$ but the statistical support was weaker than in hyperopes, likely due to the difference in the prevalence of strabismus between these subsamples $(0.8 \%$ in emmetropes vs. $5.0 \%$ in hyperopes). The stratified analysis therefore provided further evidence that the association between rs75078292 and strabismus was not driven by refractive error. 
Fig. 3 Manhattan plot for selfreported strabismus GWAS. The $y$ axis indicates minus $\log _{10} p$ values and the $x$ axis genomic position. The red and blue horizontal lines correspond to $p$ values of $5.0 \mathrm{E}-08$ and $1.0 \mathrm{E}-05$, respectively

Fig. 4 Regional association plot for self-reported strabismus in the region of strongest association, before (a) and after (b) conditioning on the lead variant


\section{Functional evaluation of the 17q25.3 locus}

Notably, the lead variant at the strabismus locus, rs75078292, is in very tight $\mathrm{LD}\left(r^{2}=0.98\right)$ with non-synonymous variant rs6420484, which introduces a tyrosine residue in place of an evolutionarily conserved cysteine at position 177 of TSPAN10, the gene encoding tetraspanin-10 (Online Resource 4). The C177Y substitution would be expected to have adverse functional consequences, given its scaled CADD score of 16.3 (Table 4). 

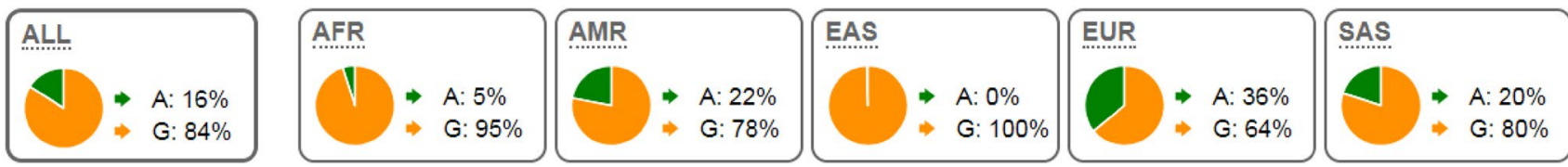

Fig. 5 Allele frequency spectrum and of lead GWAS variant across ancestry groups. Allele frequencies for rs75078292. Abbreviations: AFR, Africans; AMR, Native Americans; EAS, East Asians; EUR,

Europeans; SAS, South Asians). The results were obtained from Ensembl (Yates et al. 2016) for participants of the 1000-genomes project (The 1000 Genomes Project Consortium 2012)

Table 2 Comparison of modes of inheritance of lead variant at the NPLOC4-TSPAN10-PDE6G locus for association with self-reported strabismus in UK Biobank participants $(n=66,694)$

\begin{tabular}{|c|c|c|c|c|c|c|c|c|c|}
\hline \multirow[t]{2}{*}{ Covariates included in model } & \multicolumn{3}{|c|}{ Additive model } & \multicolumn{3}{|c|}{ Dominant model } & \multicolumn{3}{|c|}{ Recessive model } \\
\hline & OR & $95 \% \mathrm{CI}$ & $p$ value & OR & $95 \% \mathrm{CI}$ & $p$ value & OR & $95 \% \mathrm{CI}$ & $p$ value \\
\hline Baseline & 1.24 & $1.14-1.34$ & $8.92 \mathrm{E}-08$ & 1.16 & $1.04-1.29$ & $1.03 \mathrm{E}-02$ & 1.62 & $1.41-1.86$ & $1.01 \mathrm{E}-11$ \\
\hline Baseline + amblyopia status & 1.16 & $1.08-1.26$ & $1.70 \mathrm{E}-04$ & 1.11 & $0.99-1.24$ & $7.66 \mathrm{E}-02$ & 1.42 & $1.23-1.64$ & $2.16 \mathrm{E}-06$ \\
\hline Baseline + refractive error & 1.26 & $1.16-1.36$ & $2.24 \mathrm{E}-08$ & 1.19 & $1.06-1.33$ & $3.04 \mathrm{E}-03$ & 1.64 & $1.42-1.89$ & $1.61 \mathrm{E}-11$ \\
\hline Baseline + anisometropia & 1.23 & $1.13-1.33$ & $2.97 \mathrm{E}-07$ & 1.16 & $1.03-1.29$ & $1.13 \mathrm{E}-02$ & 1.58 & $1.37-1.82$ & $1.65 \mathrm{E}-10$ \\
\hline Baseline + astigmatism & 1.21 & $1.12-1.31$ & $2.01 \mathrm{E}-06$ & 1.13 & $1.01-1.26$ & $3.45 \mathrm{E}-02$ & 1.57 & $1.36-1.81$ & $4.47 \mathrm{E}-10$ \\
\hline Baseline + myopia status & 1.25 & $1.16-1.35$ & $1.71 \mathrm{E}-08$ & 1.17 & $1.05-1.31$ & $5.39 \mathrm{E}-03$ & 1.66 & $1.44-1.90$ & $1.25 \mathrm{E}-12$ \\
\hline Baseline + anisometropia status & 1.22 & $1.13-1.32$ & $7.28 \mathrm{E}-07$ & 1.15 & $1.03-1.29$ & $1.38 \mathrm{E}-02$ & 1.55 & $1.35-1.78$ & $8.50 \mathrm{E}-10$ \\
\hline Baseline + astigmatism status & 1.22 & $1.12-1.32$ & $1.07 \mathrm{E}-06$ & 1.14 & $1.02-1.28$ & $2.23 \mathrm{E}-02$ & 1.57 & $1.36-1.81$ & $3.77 \mathrm{E}-10$ \\
\hline
\end{tabular}

Baseline model included covariates: age, sex, first 10 PC, genotyping array; covariate definitions. Refractive error $=$ mean spherical equivalent refractive error in diopters averaged between the two eyes; anisometropia=anisometropia in diopters coded as a continuous variable; astigmatism $=$ refractive astigmatism in diopters averaged between the two eyes; myopia status = binary variable coded as zero unless refractive error averaged between the two eyes $\leq-0.5 \mathrm{D}$; anisometropia status $=$ binary variable coded as zero unless anisometropia $\geq 1.00 \mathrm{D}$; astigmatism status $=$ binary variable coded as zero unless refractive astigmatism averaged between the two eyes $\geq 1.00 \mathrm{D}$

Table 3 Association of lead variant for strabismus at the NPLOC4-TSPAN10-PDE6G locus with self-reported strabismus after stratifying by refractive status

\begin{tabular}{|c|c|c|c|c|c|c|c|c|}
\hline \multirow[t]{2}{*}{ Stratum } & \multirow[t]{2}{*}{ Covariates } & \multirow[t]{2}{*}{ Cases/controls } & \multicolumn{3}{|c|}{ Additive model } & \multicolumn{3}{|c|}{ Recessive model } \\
\hline & & & OR & $95 \%$ CI & $p$ value & OR & $95 \%$ CI & $p$ value \\
\hline Myopes & Baseline & $162 / 20,473$ & 1.15 & $0.93-1.44$ & $1.99 \mathrm{E}-01$ & 1.11 & $0.73-1.70$ & $6.19 \mathrm{E}-01$ \\
\hline Emmetropes & Baseline & $104 / 17,705$ & 1.22 & $0.93-1.60$ & $1.51 \mathrm{E}-01$ & 1.88 & $1.19-2.99$ & $7.24 \mathrm{E}-03$ \\
\hline Hyperopes & Baseline & $807 / 16,217$ & 1.28 & $1.15-1.42$ & $3.19 \mathrm{E}-06$ & 1.64 & $1.35-1.99$ & $8.30 \mathrm{E}-07$ \\
\hline Myopes & Adjusted $^{\mathrm{a}}$ & $162 / 20,473$ & 1.15 & $0.92-1.44$ & $2.09 \mathrm{E}-01$ & 1.11 & $0.72-1.70$ & $6.44 \mathrm{E}-01$ \\
\hline Emmetropes & Adjusted $^{\mathrm{a}}$ & $104 / 17,705$ & 1.18 & $0.89-1.55$ & $2.51 \mathrm{E}-01$ & 1.77 & $1.10-2.84$ & $1.96 \mathrm{E}-02$ \\
\hline Hyperopes & Adjusted $^{\mathrm{a}}$ & $807 / 16,217$ & 1.18 & $1.06-1.31$ & $2.26 \mathrm{E}-03$ & 1.47 & $1.20-1.80$ & $1.86 \mathrm{E}-04$ \\
\hline
\end{tabular}

Participants were classified into refractive error categories based on their spherical equivalent refractive error in each eye meeting the following criteria: myopes: $\leq-0.5 \mathrm{D}$; emmetropes: $>-0.5 \mathrm{D}$ and $\leq+1.0 \mathrm{D}$; hyperopes: $>+1.0$ D. Note that participants whose two eyes were not classified into the same group (e.g. one eye myopic and one eye emmetropic) were excluded. The baseline covariates age, sex, genotyping array, and the first 10 PCs were included in all models

${ }^{a}$ Adjusted for baseline covariates plus presence/absence of amblyopia, presence/absence of strabismus, and anisometropia (D)
The strabismus lead variant rs75078292 and the C177Yassociated variant rs6420484 are also in extremely high LD with an indel, rs397693108, also predicted to have functional consequences (CADD score 16.1). The rs397693108 indel introduces a 4-bp deletion in the mRNA for 2 isoforms of tetraspanin-10, which would result in a frameshift in the coding region. For a third isoform, the indel is predicted to result in nonsense-mediated decay (NMD) of the mRNA. The risk alleles of the C177Y-associated variant rs6420484 and indel rs397693108 have the same MAF, which given their high LD $\left(r^{2}=0.98\right)$ implies they occur on the same haplotype. CADD scores for other variants in LD with the lead GWAS variant were lower (Table 4). 
Table 4 CADD scores and eQTL effects for TSPAN10 gene expression in cerebellum for variants surpassing the genome-wide significance threshold and in very high $\operatorname{LD}\left(r^{2}>0.95\right)$ with the lead variant at the locus

\begin{tabular}{|c|c|c|c|c|c|c|c|c|c|c|}
\hline SNP & CHR & $\mathrm{BP}$ & REF & ALT & GWAS OR & GWAS $p$ value & MAF & $\begin{array}{l}\text { Scaled } \\
\text { CADD } \\
\text { score }\end{array}$ & eQTL $p$ value & eQTL effect size \\
\hline rs6420484 & 17 & 79612397 & A & G & 1.21 & $8.24 \mathrm{E}-10$ & 0.35 & 16.29 & $3.30 \mathrm{E}-21$ & 0.84 \\
\hline rs397693108 & 17 & 79614932 & TTAAC & $\mathrm{T}$ & 1.21 & $6.90 \mathrm{E}-10$ & 0.35 & 16.08 & $3.30 \mathrm{E}-21$ & 0.84 \\
\hline rs9895741 & 17 & 79603831 & A & G & 1.21 & $1.43 \mathrm{E}-09$ & 0.35 & 10.84 & $3.30 \mathrm{E}-21$ & 0.84 \\
\hline rs9747347 & 17 & 79606820 & $\mathrm{~T}$ & $\mathrm{C}$ & 1.21 & $1.27 \mathrm{E}-09$ & 0.35 & 7.09 & $1.80 \mathrm{E}-21$ & 0.84 \\
\hline rs7405453 & 17 & 79615572 & A & G & 1.21 & $5.02 \mathrm{E}-10$ & 0.35 & 6.92 & $3.30 \mathrm{E}-21$ & 0.84 \\
\hline rs62075722 & 17 & 79611271 & A & G & 1.21 & $8.48 \mathrm{E}-10$ & 0.35 & 6.29 & $3.30 \mathrm{E}-21$ & 0.84 \\
\hline rs67050149 & 17 & 79557043 & $\mathrm{C}$ & A & 0.83 & $1.25 \mathrm{E}-09$ & 0.35 & 5.98 & $3.30 \mathrm{E}-21$ & -0.84 \\
\hline rs11656126 & 17 & 79564542 & G & A & 0.83 & $7.96 \mathrm{E}-10$ & 0.35 & 5.98 & $3.00 \mathrm{E}-21$ & -0.84 \\
\hline rs8081701 & 17 & 79599441 & $\mathrm{~T}$ & $\mathrm{C}$ & 1.21 & $1.47 \mathrm{E}-09$ & 0.35 & 2.41 & $5.40 \mathrm{E}-21$ & 0.86 \\
\hline rs11650127 & 17 & 79572253 & G & A & 0.83 & $6.00 \mathrm{E}-10$ & 0.35 & 1.85 & $1.70 \mathrm{E}-20$ & -0.84 \\
\hline rs71373084 & 17 & 79564930 & $\mathrm{C}$ & G & 0.83 & $8.03 \mathrm{E}-10$ & 0.35 & 1.65 & $3.40 \mathrm{E}-21$ & -0.84 \\
\hline rs9905786 & 17 & 79602063 & G & $\mathrm{T}$ & 1.21 & $1.44 \mathrm{E}-09$ & 0.35 & 1.08 & $7.20 \mathrm{E}-22$ & 0.86 \\
\hline rs112364254 & 17 & 79578287 & G & A & 0.82 & $4.93 \mathrm{E}-10$ & 0.35 & 1.04 & $2.70 \mathrm{E}-20$ & -0.82 \\
\hline rs7503894 & 17 & 79583473 & $\mathrm{~T}$ & $\mathrm{C}$ & 1.21 & $5.94 \mathrm{E}-10$ & 0.35 & 0.64 & $3.30 \mathrm{E}-21$ & 0.84 \\
\hline rs12953229 & 17 & 79554271 & G & A & 0.83 & $9.50 \mathrm{E}-10$ & 0.35 & 0.48 & $3.30 \mathrm{E}-21$ & -0.84 \\
\hline rs75078292 & 17 & 79585492 & A & G & 1.21 & $7.95 \mathrm{E}-10$ & 0.35 & 0.38 & $3.20 \mathrm{E}-21$ & 0.84 \\
\hline rs62075723 & 17 & 79611326 & G & A & 1.21 & $5.91 \mathrm{E}-10$ & 0.35 & 0.15 & $3.30 \mathrm{E}-21$ & 0.84 \\
\hline rs34635363 & 17 & 79549250 & G & A & 0.83 & $9.34 \mathrm{E}-10$ & 0.35 & 0.13 & $3.30 \mathrm{E}-21$ & -0.84 \\
\hline rs12948708 & 17 & 79558741 & G & A & 0.83 & $9.34 \mathrm{E}-10$ & 0.35 & 0.02 & $3.30 \mathrm{E}-21$ & -0.84 \\
\hline
\end{tabular}

To search for further evidence of a functional effect of the frameshift/NMD-causing indel rs397693108, we examined TSPAN10 gene expression in tissue samples analysed by the GTEx consortium (The GTEx Consortium 2015). Although the tissues potentially most relevant to strabismus development, such as extraocular muscle, retina and visual cortex, were not amongst the 53 tissues assessed by the GTEx Consortium, the regulation of gene expression is often conserved across tissue types and therefore can still be highly informative (Pierson et al. 2015). In cerebellum and human cerebellar hemisphere the strabismus-associated risk allele was strongly associated with reduced expression of TSPAN10 ( $p=1.30 \mathrm{E}-10$ and $p=8.10 \mathrm{E}-12$, respectively; Fig. 6a, b) consistent with degradation of an mRNA isoform via NMD. Furthermore, there was evidence for cis-eQTL effects at two other genes. In testis, the risk allele was associated with reduced phosphodiesterase $6 \mathrm{G}$ gene $(P D E 6 G)$ expression ( $p=5.82 \mathrm{E}-06$; Fig. $6 \mathrm{c})$, while in thyroid, it was associated with expression of ADP ribosylation factor like GTPase 16 (ARL16) mRNA levels ( $p=2.10 \mathrm{E}-06$; Fig. 6d). In testis, rs397693108 was the 47th-ranked eSNP associated with $P D E 6 G$ expression; however, it had an almost 1.6-fold lower effect than the top-ranked variant located $15 \mathrm{~kb}$ distant (rs11150804, $p=1.80-41$ ). Like PDE6G, the ARL16 gene encoding ADP ribosylation factor like GTPase 16 is located nearby to NPLOC4 and TSPAN1O (Fig. 4). In thyroid, rs397693108 was the 232nd ranked eSNP associated with ARL16 expression, and had a more than fourfold lower effect than the top-ranked variant situated $34.5 \mathrm{~kb}$ away (rs7503637, $p=5.50 \mathrm{E}-96$ ). By contrast, rs397693108 was the 15th highest-ranked TSPAN10 eQTL in cerebellum and had an effect size equal to the top-ranked variant (rs9905786, $p=7.20 \mathrm{E}-122$ ). In cerebellar hemisphere tissue rs397693108 was the 63rd-ranked TSPAN10 eQTL.

Structural variants (SVs) are also known to regulate gene expression, including at the 17q25.3 locus where Chiang et al. (2017) found evidence for co-regulation of TSPAN10 gene expression by SVs and SNPs. Thus, in summary, there was strong evidence that the causal variant underlying the association with strabismus acts as a cis-eQTL for TSPAN10 in neural tissue, and for PDE6G and ARL16 in certain other tissues. Of these three eGenes, the evidence suggested TSPAN10 as the most likely causal eGene. However, since gene expression can be highly tissue-specific, further functional studies will be required to examine if eQTL effects are important in the mechanism by which this locus influences strabismus development.

\section{Independent replication in a clinician-diagnosed sample of children}

We sought independent replication of the findings from UK Biobank in a sample of 7-year-old children $(n=5200)$ from the ALSPAC cohort who were clinically assessed for 

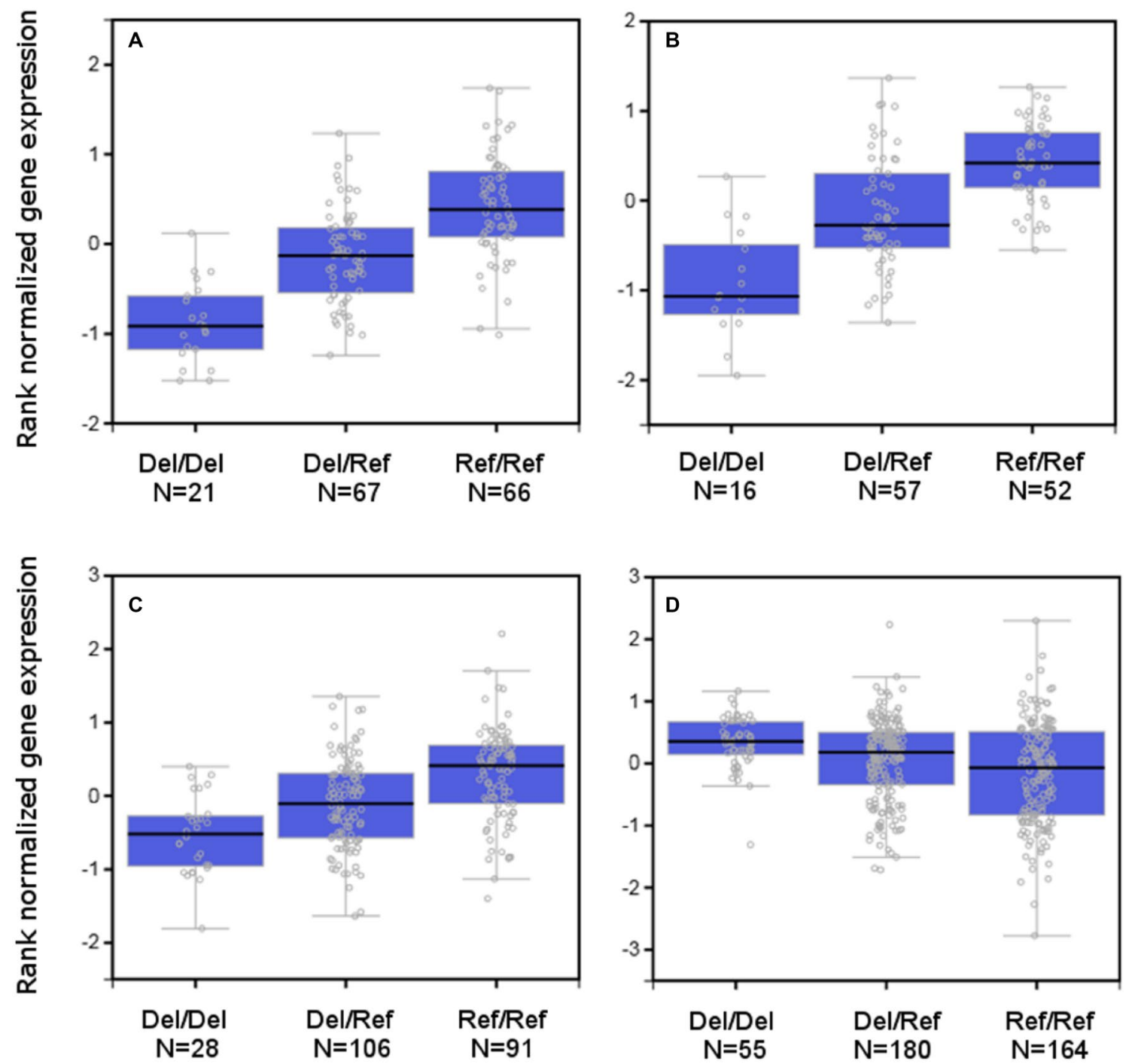

Fig. 6 Strabismus-associated indel rs397693108 is an eQTL. Gene expression levels of tetraspanin-10 mRNA in cerebellum (a) and human cerebellar hemisphere (b), and of phosphodiesterase $6 \mathrm{G}$ in testis (c), and of ADP ribosylation factor like GTPase 16 in thyroid (d) for individuals with the indicated rs397693108 genotype (Ref

allele $=$ TTAAC, Del allele $=\mathrm{T}$ ). Numbers indicate the number of donors for each genotype category. Boxes depict the first and third quartile; whiskers extend to the furthest data point up to a maximum of 1.5 times the height of the box

strabismus by an experienced orthoptist. The results are presented in Table 5. The association with strabismus was replicated in the clinician-diagnosed sample of children (Table 5). For any manifest strabismus, the $\mathrm{OR}=1.44(95 \%$ CI $1.11-1.88, p=0.007)$ and $\mathrm{OR}=1.85$ (95\% CI 1.16-2.95, $p=0.009$ ) for additive and recessive models, respectively, were consistent with UK Biobank in terms of risk allele, effect size and likely mode of inheritance. Adjusting for amblyopia in these analyses had negligible impact (Table 5). It was notable that the association with strabismus appeared to be restricted to exotropia (divergent strabismus). However, some of the children may have had exotropia resulting from surgery to correct esotropia, hence this latter result should be interpreted with caution (especially in view of the small number of only 28 children with exotropia in the ALSPAC sample).

\section{Tissue distribution of TSPAN-10 in mouse retina}

In the mouse retina, tetraspanin-10 co-localised with Peanut Agglutinin Lectin (PNA), which is a marker of cone photoreceptor inner and outer segments. There was no colocalisation with rhodopsin, a rod photoreceptor marker (Fig. 7). NPL4 homolog, ubiquitin recognition factor (NPLOC4) co-localised with G Protein subunit alpha O1 (G0alpha), which is a marker of ON bipolar cells, but not 
Table 5 Association of amblyopia and strabismus with lead GWAS variant in ALSPAC replication sample

\begin{tabular}{|c|c|c|c|c|c|c|}
\hline \multirow[t]{2}{*}{ Phenotype } & \multirow{2}{*}{$\begin{array}{l}\text { Sample size } \\
\text { cases/con- } \\
\text { trols }\end{array}$} & \multirow[t]{2}{*}{ Inheritance } & \multicolumn{2}{|l|}{ Baseline model } & \multicolumn{2}{|l|}{ Adjusted model } \\
\hline & & & OR $(95 \%$ CI $)$ & $p$ value & OR (95\% CI) & $p$ value \\
\hline \multirow[t]{2}{*}{ History of strabismus } & \multirow[t]{2}{*}{$145 / 5055$} & Additive & $1.36(1.07-1.73)$ & 0.011 & $1.34(1.04-1.73)$ & 0.026 \\
\hline & & Recessive & $1.72(1.12-2.63)$ & 0.013 & $1.58(0.98-2.55)$ & 0.061 \\
\hline \multirow[t]{2}{*}{ Manifest strabismus } & \multirow[t]{2}{*}{$116 / 5084$} & Additive & $1.44(1.11-1.88)$ & 0.007 & $1.43(1.07-1.92)$ & 0.016 \\
\hline & & Recessive & $1.85(1.16-2.95)$ & 0.009 & $1.72(1.00-2.95)$ & 0.050 \\
\hline \multirow[t]{2}{*}{ 'Esotropia' } & \multirow[t]{2}{*}{$143 / 5057$} & Additive & $1.08(0.85-1.38)$ & 0.542 & $1.02(0.78-1.32)$ & 0.910 \\
\hline & & Recessive & $1.20(0.74-1.95)$ & 0.450 & $1.00(0.59-1.72)$ & 0.989 \\
\hline \multirow[t]{2}{*}{ 'Exotropia' } & \multirow[t]{2}{*}{$28 / 5172$} & Additive & $1.76(1.04-2.99)$ & 0.035 & $1.73(1.02-2.93)$ & 0.040 \\
\hline & & Recessive & $2.47(1.05-5.83)$ & 0.039 & $2.37(1.00-5.63)$ & 0.051 \\
\hline \multirow[t]{2}{*}{ Amblyopia } & \multirow[t]{2}{*}{$189 / 5011$} & Additive & $1.19(0.97-1.48)$ & 0.100 & $0.97(0.77-1.23)$ & 0.789 \\
\hline & & Recessive & $1.36(0.91-2.02)$ & 0.135 & $1.10(0.69-1.74)$ & 0.690 \\
\hline
\end{tabular}

Baseline model adjusted for sex. Adjusted amblyopia analysis: adjusted for sex and strabismus. Adjusted strabismus analyses: adjusted for sex and amblyopia

Note 'Esotropia' includes large ( $>10 \mathrm{pd}$ ) esophoria as well as manifest esotropia and 'Exotropia' similarly includes large ( $>15 \mathrm{pd}$ ) exophoria as well as manifest exotropia, hence $N$ of esotropia + exotropia is greater than for manifest strabismus, which includes only cases manifest on the day of examination

Phenotypes were ascertained in children at age of 7 years with Protein kinase-C alpha (PKC), a marker of rod bipolar cells (Fig. 8). This suggested TSPAN10 is expressed in cone photoreceptors and NPLOC4 in ON-cone bipolar cells in the mouse retina. Previous studies have shown that phosphodiesterase 6G (PDE6G) is expressed in both rod and cone photoreceptors (Dvir et al. 2010).

\section{Discussion}

These results provide strong evidence that a commonly occurring polymorphism situated within a high-LD cluster of approximately 20 variants on chromosome 17q25.3 contributes to susceptibility to strabismus. This association with strabismus was independent of the previously documented association with myopia at this locus, since the degree of association with strabismus was minimally attenuated by
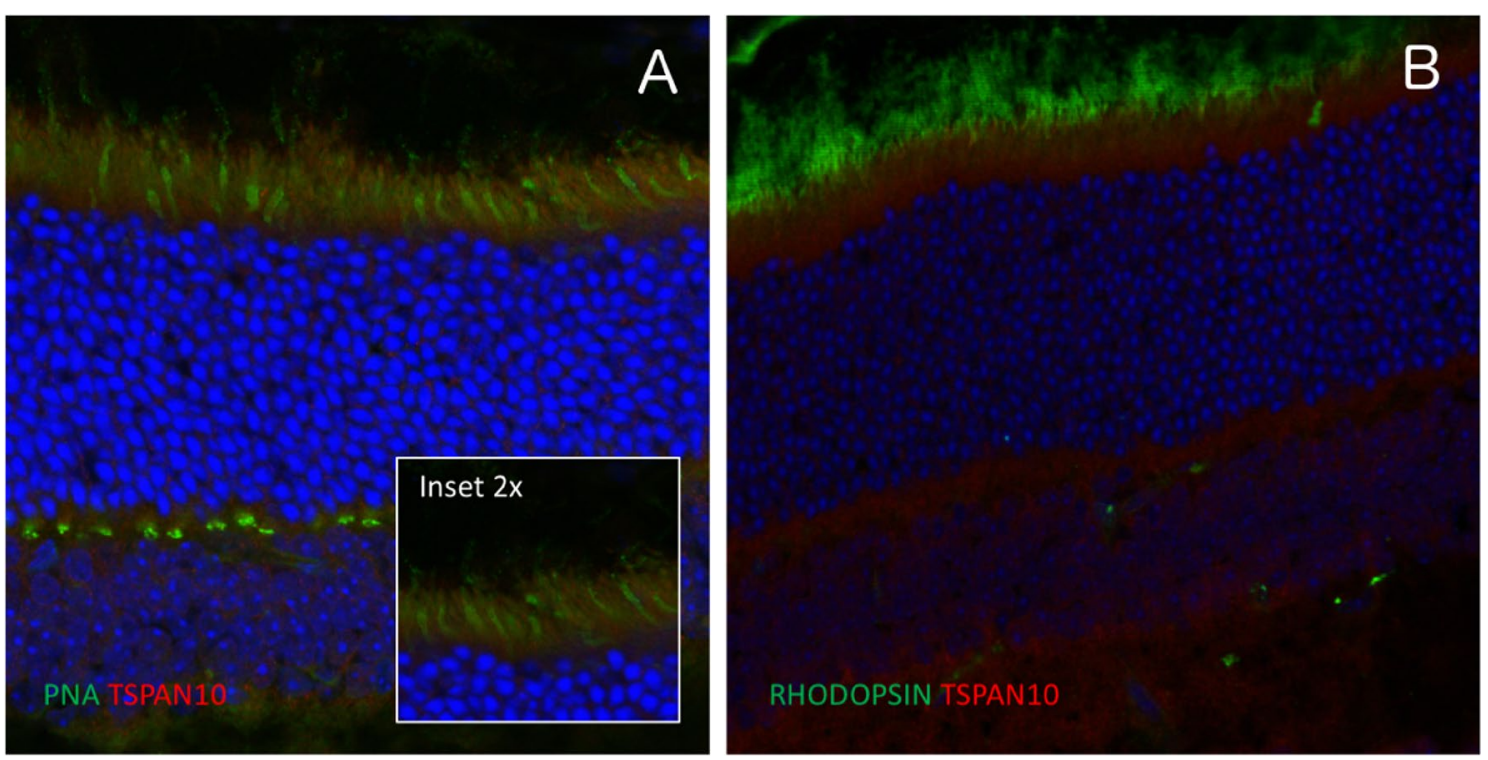

Fig. 7 Immuno-localisation of tetraspanin-10 in the mouse retina. a Tetraspanin-10 co-localised with Peanut Agglutinin Lectin (PNA), a cone photoreceptor inner and outer segment marker. b Tetraspanin-10 did not co-localise with rhodopsin, a rod photoreceptor marker 


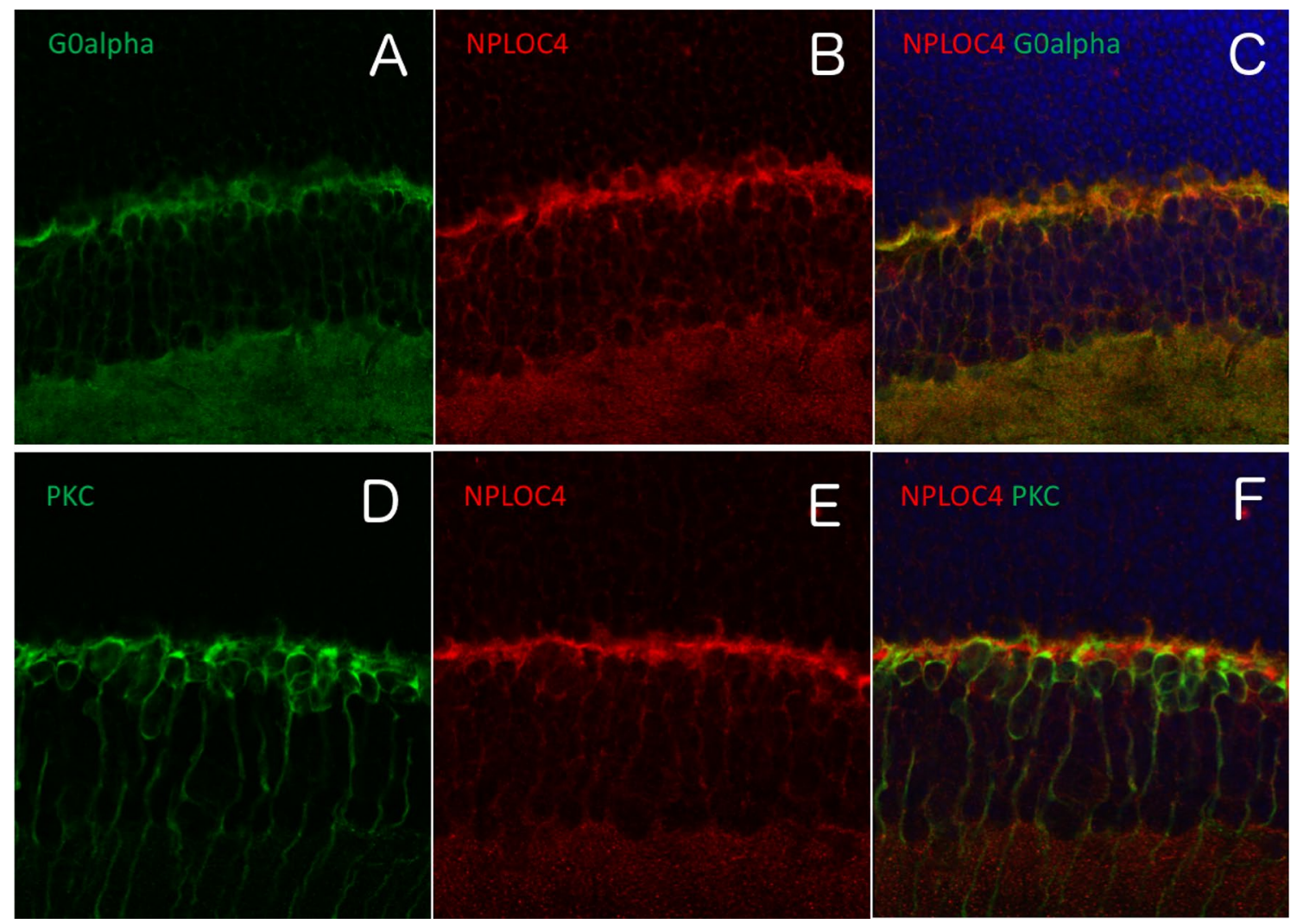

Fig. 8 Immuno-localisation of NPL4 homolog, ubiquitin recognition factor (NPLOC4) in the mouse retina. a-c NPLOC4 co-localised with G0alpha, which a marker for the dendritic tips of ON bipolar

statistical adjustment for refractive error, myopia status, or anisometropia. These findings suggest a new avenue for strabismus research; namely, to discover how and why gene(s) at the NPLOC4-TSPAN10-PDE6G locus influence the visual system's response to visual experience in childhood. Our results add to the evidence from the GWAS carried out by Shaaban et al. (2018), that commonly occurring variants contribute to susceptibility for strabismus. Notably, the lead variant (rs2244352) associated with non-accommodative esotropia in the Shaaban et al. GWAS was not associated with self-reported strabismus in the current study, suggesting the possibility that the association with rs2244352 is specific to non-accommodative esotropia rather than strabismus more generally.

We reasoned that three criteria should be met in order for a case-control GWAS to detect genetic variants associated with a trait-of-interest. First, the trait must have a genetic component. Second, the sample of 'controls' should contain relatively few individuals truly affected by the disorder, i.e. a low proportion of misclassified cases. Third, the sample of 'cases' must be enriched for individuals truly affected by the disorder. Regarding our first criterion, recent progress in identifying mutations causing strabismus (Maconachie interneurons. d-f NPLOC4 did not co-localise with protein kinase-C (PKC), a marker for rod bipolar interneurons including their dendrites

et al. 2013; Ye et al. 2014; Kruger et al. 2013; Shaaban et al. 2018) provided support for the hypothesis that susceptibility to strabismus has a genetic component. In their pioneering study employing a set of shared controls, the Wellcome Trust Case Control Consortium (2007) carried out simulations demonstrating that statistical power was minimally reduced if fewer than 5\% of 'controls' were actually misclassified/undiagnosed cases. Given the known prevalence of strabismus in the population (2-4\%), selecting controls as participants who did not self-report strabismus was therefore considered an effective approach for meeting our second criterion. To meet our third criterion we sought to confirm that the group of UK Biobank participants who self-reported strabismus was enriched for people with 'true' strabismus. To do this, we analysed the level of comorbid traits such as self-reported amblyopia, anisometropia and asymmetric VA in self-reported strabismus cases and controls, which demonstrated convincingly that the comorbidities were indeed over-represented amongst cases (Table 1; Fig. 2). Most importantly, we verified that the association with strabismus was replicated in an independent cohort of 7-year-old children with clinician-diagnosed strabismus $(\mathrm{OR}=1.85$, 95\% CI 1.16-2.95). 
There was suggestive evidence that the association with strabismus was restricted to non-myopic individuals, andcounter-intuitively - to individuals with exotropia rather than esotropia. To further dissect these inter-relationships will require the collection and genotyping of large case-control samples, or Biobank-scale cohorts, of individuals with clinician-diagnosed strabismus. In view of the worldwide differences in allele frequency of the lead variant at the NPLOC4-TSPAN10-PDE6G locus (Fig. 5), GWAS analyses in other ancestry groups should prove fruitful.

Genetic variants at the NPLOC4-TSPAN10-PDE6G locus have been associated with a diverse range of ocular phenotypes. As well as the previously mentioned association of variants at the novel strabismus locus being associated with spherical refractive error, Shah et al. (2018) recently reported that these variants were also associated with corneal and refractive astigmatism (independently of spherical refractive error), while Gao et al. (2019) reported that rs7405453, which is in perfect LD with the lead strabismus variant rs75078292 $\left(r^{2}=1.0\right)$ was associated with macular thickness. Two SNPs within NPLOC4 (rs6565597 and rs9894429) are associated with age-related macular degeneration (AMD) and eye colour, respectively (Fritsche et al. 2015; Liu et al. 2010). Missense variant rs201259422, which is located only $2.5 \mathrm{~kb}$ from the TSPAN10 C177Yassociated variant and introduces a D266N amino acid substitution in TSPAN10, is associated with microvascular (central retinal venule) diameter in human retina (Jensen et al. 2016). Given the association of variants at the NPLOC4-TSPAN10-PDE6G locus and AMD, we carried out a sensitivity analysis examining the association between strabismus and rs75078292 after excluding UK Biobank participants with self-reported AMD. The results were minimally affected (Online Resource 5).

Under the most parsimonious inheritance model-recessive inheritance - the magnitude of the association with strabismus $(\mathrm{OR} \approx 1.4-1.8)$ is much larger than those typically reported in GWAS studies of complex traits (MacArthur et al. 2017). In view of the wide range of adverse effects associated with the NPLOC4-TSPAN10-PDE6G locus, it is conceivable that the locus has compensatory beneficial effects on other traits.

In silico functional prediction suggested TSPAN10 as the most likely candidate gene at the locus. The tetraspanin gene family encodes a series of glycosylated, 4-pass transmembrane proteins that feature 1 short and 1 long extracellular loop. Through multiple protein-protein interactions involving other tetraspanins, integrins, signalling receptors, immunoglobulins and proteolytic enzymes, tetraspanins are instrumental in forming membrane microdomains with specific functions (Seipold and Saftig 2016). Mutations in PRPH2 (also known as TSPAN22 or RDS) and in ROM1 (also known as TSPAN23), which function together in stabilising the structure of photoreceptor outer segment discs, can cause retinitis pigmentosa (Kajiwara et al. 1994), while mutations in TSPAN12 can cause familial exudative vitreo-retinopathy type 5 (EVR5) (Poulter et al. 2010; Nikopoulos et al. 2010). Tetraspanin-10, which was first detected in a cDNA library from retinal pigment epithelium/choroid and given the name oculospanin (Wistow et al. 2002), is part of the TSpanC8 subfamily (tetraspanins with 8 cysteine residues in their large extracellular domain). TSpanC8 tetraspanins are implicated in the trafficking of ADAM10 and cleavage of Notch, prior to its further processing by $\gamma$-secretase (Dornier et al. 2012). Targets of $\gamma$-secretase include (Wakabayashi et al. 2009) APP (amyloid precursor protein) and APLP-2 (amyloid beta precursor-like protein-2); the latter protein has been linked to myopia development through an interaction with time spent reading (Tkatchenko et al. 2015). The precise roles of tetraspanin-10 in neuronal tissues such as cranial nerves, retina and brain are not clear. Notably, the cysteine at position 177 of TSPAN10 affected by rs6420484 is not one of the 8 cysteines in its large extracellular loop, suggesting that the SNP would not directly impair cleavage activity. The NPLOC4 gene encodes NPL4 homolog, ubiquitin recognition factor. Little is known of the physiological role of this protein, although its predicted functions include metal ion binding, protein binding, and ubiquitin binding, suggesting a role in ubiquitin-dependent catabolism or endoplasmic reticulum/Golgi organisation. PDE6G codes for the (inhibitory) $\gamma$-subunit of cGMP-phosphodiesterase. As well as its well-known role in rod phototransduction, this protein regulates MAPK (mitogen-activated protein kinase) signalling via GRK2 (G protein-coupled receptor kinase 2) (Wan et al. 2003). Mutations in PDE6G are a rare cause of autosomal recessive retinitis pigmentosa (Dvir et al. 2010; Tsang et al. 1996).

In summary, a genome-wide association study for selfreported strabismus in a large sample of unrelated European individuals identified a single, novel locus associated with this trait.

The association with strabismus was replicated in a sample of 7-year-old children with clinician-diagnosed strabismus ( $\mathrm{OR}=1.8, p=0.009)$. The strongest candidate functional variants were a non-synonymous SNP, rs6420484, which introduces a C177Y substitution in the TSPAN10 gene, and a TSPAN10-frameshift-inducing 4-bp indel, rs397693108. These variants were associated with reduced TSPAN10 gene expression in brain tissues, although such eQTL effects were also observed for the adjacent genes PDE6G and ARL16, suggesting that the risk of strabismus could be mediated through any one or more of these genes. The identification of a common polymorphism conferring susceptibility to strabismus, opens a new avenue for research to understand the causal mechanisms responsible. 
Acknowledgements We are grateful to all of the participants for making this research possible. UK Biobank: this research has been conducted using the UK Biobank Resource (application \#17351). UK Biobank was established by the Wellcome Trust; the UK Medical Research Council; the Department for Health (London, UK); Scottish Government (Edinburgh, UK); and the Northwest Regional Development Agency (Warrington, UK). It also received funding from the Welsh Assembly Government (Cardiff, UK); the British Heart Foundation; and Diabetes UK. Collection of eye and vision data was supported by The Department for Health through an award made by the NIHR to the Biomedical Research Centre at Moorfields Eye Hospital NHS Foundation Trust, and UCL Institute of Ophthalmology, London, United Kingdom (Grant no. BRC2_009). Additional support was provided by The Special Trustees of Moorfields Eye Hospital, London, United Kingdom (Grant no. ST 12 09). ALSPAC: we are extremely grateful to all the families who took part in this study, the midwives for their help in recruiting them, and the whole ALSPAC team, which includes interviewers, computer and laboratory technicians, clerical workers, research scientists, volunteers, managers, receptionists and nurses. Computing resources: data analysis was carried out using the RAVEN computing cluster, maintained by the ARCCA group at Cardiff University and the BLUE CRYSTAL3 computing cluster maintained by the HPC group at the University of Bristol.

Funding The work was funded by the Global Education Program of the Russian Federation government (DP), National Eye Research Centre grant SAC015 (JAG, CW), and an NIHR Senior Research Fellowship award SRF-2015-08-005 (CW). The UK Medical Research Council and Wellcome (Grant ref: 102215/2/13/2) and the University of Bristol provide core support for ALSPAC. This publication is the work of the authors and JAG and CW will serve as guarantors for the contents of this paper. A comprehensive list of grants funding is available on the ALSPAC website (http://www.bristol.ac.uk/alspac/external/documents/ grant-acknowledgements.pdf). ALSPAC GWAS data were generated by Sample Logistics and Genotyping Facilities at Wellcome Sanger Institute and LabCorp (Laboratory Corporation of America) using support from 23andMe.

\section{Compliance with ethical standards}

Conflict of interest On behalf of all authors, the corresponding author states that there is no conflict of interest.

Open Access This article is distributed under the terms of the Creative Commons Attribution 4.0 International License (http://creativeco mmons.org/licenses/by/4.0/), which permits unrestricted use, distribution, and reproduction in any medium, provided you give appropriate credit to the original author(s) and the source, provide a link to the Creative Commons license, and indicate if changes were made.

\section{References}

Atkinson J, Braddick O, Bobier B, Anker S, Ehrlich D, King J, Watson P, Moore A (1996) Two infant vision screening programmes: prediction and prevention of strabismus and amblyopia from photoand videorefractive screening. Eye 10:189-198

Bagos PG (2013) Genetic model selection in genome-wide association studies: robust methods and the use of meta-analysis. Stat Appl Genet Mol Biol 12(3):285-308

Benner C, Spencer CCA, Havulinna AS, Salomaa V, Ripatti S, Pirinen M (2016) FINEMAP: efficient variable selection using summary data from genome-wide association studies. Bioinformatics 32(10):1493-1501
Boyd A, Golding J, Macleod J, Lawlor DA, Fraser A, Henderson J, Molloy L, Ness A, Ring S et al (2013) Cohort profile: the "Children of the 90s"- the index offspring of the Avon longitudinal study of parents and children. Int J Epidemiol 42(1):111-127

Bycroft C, Freeman C, Petkova D, Band G, Elliott LT, Sharp K, Motyer A, Vukcevic D, Delaneau O et al (2018) The UK Biobank resource with deep phenotyping and genomic data. Nature 562(7726):203-209

Chang CC, Chow CC, Tellier LC, Vattikuti S, Purcell SM, Lee JJ (2015) Second-generation PLINK: rising to the challenge of larger and richer datasets. Gigascience 4:7

Chiang C, Scott AJ, Davis JR, Tsang EK, Li X, Kim Y, Hadzic T, Damani FN, Ganel L et al (2017) The impact of structural variation on human gene expression. Nat Genet 49(5):692-699

Cotter SA, Varma R, Tarczy-Hornoch K, McKean-Cowdin R, Lin J, Wen G, Wei J, Borchert M, Azen SP et al (2011) Risk factors associated with childhood strabismus: the multi-ethnic pediatric eye disease and Baltimore pediatric eye disease studies. Ophthalmol 118(11):2251-2261

Cumberland PM, Rahi JS, UK Biobank Eye \& Vision Consortium (2016) Visual function, social position, and health and life chances: the UK Biobank study. JAMA Ophthalmol 134(9):959-966

Dornier E, Coumailleau F, Ottavi JF, Moretti J, Boucheix C, Mauduit P, Schweisguth F, Rubinstein E (2012) TspanC8 tetraspanins regulate ADAM10/Kuzbanian trafficking and promote Notch activation in flies and mammals. J Cell Biol 199(3):481-496

Dvir L, Srour G, Abu-Ras R, Miller B, Shalev SA, Ben-Yosef T (2010) Autosomal-recessive early-onset retinitis pigmentosa caused by a mutation in PDE6G, the gene encoding the gamma subunit of rod cGMP phosphodiesterase. Am J Hum Genet 87(2):258-264

Fraser A, Macdonald-Wallis C, Tilling K, Boyd A, Golding J, Davey Smith G, Henderson J, Macleod J, Molloy L et al (2012) Cohort profile: the Avon longitudinal study of parents and children: ALSPAC mothers cohort. Int J Epidemiol 42(1):97-110

Fritsche LG, Igl W, Bailey JNC, Grassmann F, Sengupta S, BraggGresham JL, Burdon KP, Hebbring SJ, Wen C et al (2015) A large genome-wide association study of age-related macular degeneration highlights contributions of rare and common variants. Nat Genet 48(2):134-143

Gao XR, Huang H, Kim H (2019) Genome-wide association analyses identify 139 loci associated with macular thickness in the UK Biobank cohort. Hum Mol Genet. https://doi.org/10.1093/hmg/ ddy 1422 (in press)

Georges A, Cambisano N, Ahariz N, Karim L, Georges M (2013) A genome scan conducted in a multigenerational pedigree with convergent strabismus supports a complex genetic determinism. PLoS One 8(12):e83574

Graeber CP, Hunter DG, Engle EC (2013) The genetic basis of incomitant strabismus: consolidation of the current knowledge of the genetic foundations of disease. Semin Ophthalmol 28(5-6):427-437

Jensen RA, Sim X, Smith AV, Li X, Jakobsdóttir J, Cheng C-Y, Brody JA, Cotch MF, McKnight B et al (2016) Novel genetic loci associated with retinal microvascular diameter. Circ Cardiovasc Genet $9(1): 45-54$

Jung CC, Atan D, Ng D, Ploder L, Ross SE, Klein M, Birch DG, Diez E, McInnes RR (2015) Transcription factor PRDM8 is required for rod bipolar and type 2 OFF-cone bipolar cell survival and amacrine subtype identity. Proc Natl Acad Sci USA 112(23):E3010-E3019

Kajiwara K, Berson EL, Dryja TP (1994) Digenic retinitis-pigmentosa due to mutations at the unlinked peripherin/RDS and ROM1 loci. Science 264(5165):1604-1608

Kruger JM, Mansouri B, Cestari DM (2013) An update on the genetics of comitant strabismus. Semin Ophthalmol 28(5-6):438-441

Liu F, Wollstein A, Hysi PG, Ankra-Badu GA, Spector TD, Park D, Zhu G, Larsson M, Duffy DL et al (2010) Digital quantification of 
human eye color highlights genetic association of three new loci. PLoS Genet 6(5):e1000934

Ma C, Blackwell T, Boehnke M, Scott LJ, GoT2D Investigators, (2013) Recommended joint and meta-analysis strategies for case-control association testing of single low-count variants. Genet Epidemiol 37(6):539-550

MacArthur J, Bowler E, Cerezo M, Gil L, Hall P, Hastings E, Junkins H, McMahon A, Milano A et al (2017) The new NHGRI-EBI Catalog of published genome-wide association studies (GWAS Catalog). Nucl Acids Res 45(D1):D896-D901

Maconachie GE, Gottlob I, McLean RJ (2013) Risk factors and genetics in common comitant strabismus: a systematic review of the literature. JAMA Ophthalmol 131(9):1179-1186

McCarthy S, Das S, Kretzschmar W, Delaneau O, Wood AR, Teumer A, Kang HM, Fuchsberger C, Danecek P et al (2016) A reference panel of 64,976 haplotypes for genotype imputation. Nat Genet 48(10):1279-1283

Nikopoulos K, Gilissen C, Hoischen A, van Nouhuys CE, Boonstra FN, Blokland EA, Arts P, Wieskamp N, Strom TM et al (2010) Next-generation sequencing of a $40 \mathrm{Mb}$ linkage interval reveals TSPAN12 mutations in patients with familial exudative vitreoretinopathy. Am J Hum Genet 86(2):240-247

Parikh V, Shugart YY, Doheny KF, Zhang J, Li L, Williams J, Hayden D, Craig B, Capo H et al (2003) A strabismus susceptibility locus on chromosome 7p. Proc Natl Acad Sci USA 100(21):12283-12288

Pathai S, Cumberland PM, Rahi JS (2010) Prevalence of and early-life influences on childhood strabismus: findings from the millennium cohort study. Arch Pediatr Adolesc Med 164(3):250-257

Pickrell JK, Berisa T, Liu JZ, Segurel L, Tung JY, Hinds DA (2016) Detection and interpretation of shared genetic influences on 42 human traits. Nat Genet 48(7):709-717

Pierson E, Koller D, Battle A, Mostafavi S (2015) Sharing and specificity of co-expression networks across 35 human tissues. PLoS Comput Biol 11(5):e1004220

Poulter JA, Ali M, Gilmour DF, Rice A, Kondo H, Hayashi K, Mackey DA, Kearns LS, Ruddle JB et al (2010) Mutations in TSPAN12 cause autosomal-dominant familial exudative vitreoretinopathy. Am J Hum Genet 86(2):248-253

Qi L, Ma J, Qi Q, Hartiala J, Allayee H, Campos H (2011) A genetic risk score and risk of myocardial infarction in Hispanics. Circulation 123(4):374-380

Qin X-J, Margrain TH, To CH, Bromham N, Guggenheim JA (2005) Anisometropia is independently associated with both spherical and cylindrical ametropia. Invest Ophthalmol Vis Sci 46:4024-4031

R Development Core Team (2008) R: a language and environment for statistical computing. R Foundation for Statistical Computing, Vienna

Rice A, Jrm Nsengimana, Simmons IG, Toomes C, Hoole J, Willoughby CE, Cassidy F, Williams GA, George ND et al (2009) Replication of the recessive STBMS1 locus but with dominant inheritance. Invest Ophthalmol Vis Sci 50(7):3210-3217

Robaei D, Rose KA, Kifley A, Cosstick M, Ip JM, Mitchell P (2006) Factors associated with childhood strabismus: findings from a population-based study. Ophthalmol 113(7):1146-1153

Sanfilippo PG, Hammond CJ, Staffieri SE, Kearns LS, Melissa Liew SH, Barbour JM, Hewitt AW, Ge D, Snieder H et al (2012) Heritability of strabismus: genetic influence is specific to eso-deviation and independent of refractive error. Twin Res Hum Genet 15(5):624-630

Seipold L, Saftig P (2016) The emerging role of tetraspanins in the proteolytic processing of the amyloid precursor protein. Front Mol Neurosci 9:149

Shaaban S, MacKinnon S, Andrews C, Staffieri SE, Maconachie GDE, Chan W-M, Whitman MC, Morton SU, Yazar S et al (2018) Genome-wide association study identifies a susceptibility locus for comitant Esotropia and suggests a parent-of-origin effect. Invest Ophthalmol Vis Sci 59(10):4054-4064

Shah RL, Guggenheim JA, UK Biobank Eye and Vision Consortium (2018) Genome-wide association studies for corneal and refractive astigmatism in UK Biobank demonstrate a shared role for myopia susceptibility loci. Hum Genet 137(11):881-896

Sudlow C, Gallacher J, Allen N, Beral V, Burton P, Danesh J, Downey P, Elliott P, Green J et al (2015) UK Biobank: an open access resource for identifying the causes of a wide range of complex diseases of middle and old age. PLoS Med 12(3):e1001779

Taylor M, Simpkin AJ, Haycock PC, Dudbridge F, Zuccolo L (2016) Exploration of a polygenic risk score for alcohol consumption: a longitudinal analysis from the ALSPAC cohort. PLoS One 11(11):e0167360

Tedja MS, Wojciechowski R, Hysi PG, Eriksson N, Furlotte NA, Verhoeven VJM, Iglesias AI, Meester-Smoor MA, Tompson SW et al (2018) Genome-wide association meta-analysis highlights light-induced signaling as a driver for refractive error. Nat Genet 50:834-848

The 1000 Genomes Project Consortium (2012) An integrated map of genetic variation from 1,092 human genomes. Nature 491(7422):56-65

The GTEx Consortium (2015) The Genotype-Tissue Expression (GTEx) pilot analysis: multitissue gene regulation in humans. Science 348(6235):648-660

Tkatchenko AV, Tkatchenko TV, Guggenheim JA, Verhoeven VJ, Hysi PG, Wojciechowski R, Singh PK, Kumar A, Thinakaran G et al (2015) APLP2 regulates refractive error and myopia development in mice and humans. PLoS Genet 11(8):e1005432

Tsang SH, Gouras P, Yamashita CK, Kjeldbye H, Fisher J, Farber DB, Goff SP (1996) Retinal degeneration in mice lacking the $\gamma$ subunit of the rod cGMP phosphodiesterase. Science 272(5264):1026-1029

Wakabayashi T, Craessaerts K, Bammens L, Bentahir M, Borgions F, Herdewijn P, Staes A, Timmerman E, Vandekerckhove J et al (2009) Analysis of the [gamma]-secretase interactome and validation of its association with tetraspanin-enriched microdomains. Nat Cell Biol 11(11):1340-1346

Wan KF, Sambi BS, Tate R, Waters C, Pyne NJ (2003) The inhibitory gamma subunit of the type 6 retinal cGMP phosphodiesterase functions to link c-Src and G-protein-coupled receptor kinase 2 in a signaling unit that regulates p42/p44 mitogen-activated protein kinase by epidermal growth factor. J Biol Chem 278(20):18658-18663

Wellcome Trust Case Control Consortium (2007) Genome-wide association study of 14,000 cases of seven common diseases and 3,000 shared controls. Nature 447(7145):661-678

Williams C, Northstone K, Howard M, Harvey I, Harrad RA, Sparrow JM (2008) Prevalence and risk factors for common vision problems in children: data from the ALSPAC study. Br J Ophthalmol 92(7):959-964

Wistow G, Berstein SL, Wyatt MK, Farriss RN, Behal A, Touchman J, Bouffard G, Smith D, Peterson K (2002) Expressed sequence tag analysis of human RPE/choroid for the NEIBank Project: over 6000 non-redundant transcripts, novel genes and splice variants. Mol Vis 8(21):205-220

Yates A, Akanni W, Amode MR, Barrell D, Billis K, Carvalho-Silva D, Cummins C, Clapham P, Fitzgerald S et al (2016) Ensembl 2016. Nucl Acids Res 44(D1):D710-D716

Ye XC, Pegado V, Patel MS, Wasserman WW (2014) Strabismus genetics across a spectrum of eye misalignment disorders. Clin Genet 86(2):103-111

Publisher's Note Springer Nature remains neutral with regard to jurisdictional claims in published maps and institutional affiliations. 